socio-ecological resilience. Using multiproxy records, this group of studies revealed long-term interactions between climate, human activities and ecosystem services, the presence of thresholds and early warning signals, and reference conditions for conservation. Zhang Ke from Southampton (UK) shed light on recent attempts to use paleoenvrionmental records as proxies for ecosystem services in the lower Yangtze basin (Fig. 1). The paper by Wang Rong (Southampton, UK) examined the evidence for early warning indicators of eutrophication in lake sediments from southwestern China. And Helen Shaw (Lancaster, UK) assessed paleoecological and historical contributions to understanding sustainability, resilience, and ecosystem services within traditional pastoral management. The wide range of questions that were addressed across many geographical zones, served to emphasize the growing use of paleoenvironmental archives to understand human-environment interactions. Reflections on the meeting by recipients of PAGES support gave a flavor of the intellectual atmosphere and rapport generated during the meeting. One recipient commented that the meeting was timely: taking place not only when ecosystems are increasingly threatened by rapid climate change and human activities but when scientific communities across the globe are trying to find the best possible approaches to mitigate these effects. This meeting was a significant step toward our efforts for a comprehensive understanding of the human-climate-ecosystem interactions during the $21^{\text {st }}$ century that help promote societal and ecosystem resilience against future climate change. The use of a range of proxy indicators to understand ecosystem response to climate and human drivers can help us develop not only management tools but also our theoretical understanding of ecosystem responses to multiple and complex forcing.

\section{References}

Dearing Jet al. (2012) PNAS Plus 109(18): E1111-1120

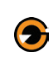

\title{
Report on the PAGES/CLIVAR Intersection Panel Meeting
}

\section{WCRP, Denver, USA, 28 October 2011}

Gavin A. Schmidt ${ }^{1}$ and Valérie Masson-Delmotte ${ }^{2}$, Co-Chairs

'NASA Goddard Institute for Space Studies, New York, USA; gavin.a.schmidt@nasa.gov

${ }^{2}$ Institut Pierre Simon Laplace and Laboratoire des Sciences du Climat et de l'Environnement, Gif-sur-Yvette, France

Sine ince 1999, the PAGES/CLIVAR (Climate Variability and Predictability) Intersection Panel (www.clivar.org/organization/pages) has focused on benefitting from the combined expertise and insights of scientists working on modern climate observations and processes and those collecting and interpreting paleoclimate records.

The World Climate Research Programme (WCRP) Open Science Meeting in Denver, USA, in October 2011, featured many examples of paleoclimate information being used to extend the instrumental record, evaluate climate models, and place modern changes in a longer-term context. It was therefore an excellent backdrop for the annual committee meeting of the Intersection Panel.

Our Panel meeting was dedicated to finding the best strategies for boosting collaborations across the WCRP "seamless" community (not just CLIVAR). Panel member rotation was addressed with an objective of ensuring a wide range of interests and geographical spread in new members. This selection is now underway, and any readers interested in joining the panel are encouraged to contact the chairs.

Links with other panels and working groups were also an important topic of discussion. Representatives from the Global Monsoon working group, CLIVAR Atlantic panel, and others expressed great interest in increasing the paleoclimate component in their discussions and projects. In particular, the PAGES Ocean2K synthesis project (www.pagesigbp.org/workinggroups/ocean2k) (motivated by our Panel thanks to CLIVAR Scientific Steering Committee inputs) that focuses on bringing together highresolution ocean proxy data for the last two millennia was highlighted as an important bridge to the observational oceanographic community. The Panel has instituted a new mailing list (clivarpages-open@clivar.org) that we hope will be a resource for notifications of cross-cutting projects and workshops, and a platform for ideas on how to engage wider community participation. To subscribe, please go to www.clivar.org/ clivarpages-mailing-list.

The centerpiece of discussion at the meeting was the Coupled Model Intercomparison Project (CMIP5) and database. For the first time within the CMIP protocol, paleoclimate simulations for the Last Glacial Maximum (21 ka ago), mid-Holocene (6 ka ago) and last millennium (Paleoclimate Modelling Intercomparison Project, PMIP3) have been included alongside historical simulations for the $20^{\text {th }}$ century and future projections. This allows for a much greater analysis of whether and how paleoclimate model/data comparisons are informative of future projections.

The Panel organized a workshop focused on this topic in March 2012 (Schmidt et al., this issue), and the outcomes were presented to the wider CMIP5 community. The Panel is working to produce a refereed "white paper" on the best practices for using the PMIP3 simulations to help constrain projections. The white paper will address issues of statistical robustness, dataset synthesis, comparison strategies and quantification of uncertainties due to the model structure, data set uncertainty and forward modeling approaches.

Overall, this is an exciting time to be bringing these communities together, and the resulting new initiatives have enormous potential. 